\title{
Comparación de la concentración del factor de crecimiento transformante Beta 1 (TGF- $\beta 1$ ) en líquido sinovial de perros con y sin enfermedad degenerativa articular
}

\author{
COMPARISON OF THE CONCENTRATION OF THE TRANSFORMING GROWTH FACTOR BETA 1 \\ (TGF-ß1) IN SYNOVIAL FLUID OF DOGS WITH AND WITHOUT DEGENERATIVE JOINT DISEASE
Christof Fischer Wiethuchter ${ }^{1,3}$, Valentina Lepe ${ }^{2}$, Álvaro Opazo ${ }^{1}$, Álvaro Luzio ${ }^{1}$, Ignacio Troncoso ${ }^{1}$, Carolina Hernandez ${ }^{1}$

\section{Resumen}

La enfermedad degenerativa articular (EDA) es una patología de carácter no inflamatorio en su origen, que se caracteriza en ser progresiva, estructural y funcional. El factor de crecimiento transformante Beta 1 (TGF- $\beta 1$ ) es una citocina reguladora en la producción y depósito de matriz extracelular en varios tejidos, incluyendo el cartílago articular. El objetivo del presente estudio fue comparar las concentraciones del factor de crecimiento transformante $\beta 1$ en líquido sinovial de caninos con $(n=12)$ y $\sin \operatorname{EDA}(n=11)$, determinado mediante una prueba de ELISA. La concentración media de TGB- $\beta 1$ en perros $\sin$ EDA fue de $16.8 \pm 7.1 \mathrm{ng} / \mathrm{ml}$, mientras que en perros con EDA fue de $10.3 \pm 3.5$ $\mathrm{ng} / \mathrm{ml}(\mathrm{p}<0.05)$.

Palabras clave: factor de crecimiento transformante $\beta 1$; líquido sinovial; caninos

\section{AbSTRACT}

Degenerative joint disease (DJD) is a non-inflammatory pathology and characterized to be progressive, structural, and functional. The transforming growth factor Beta 1 (TGF- $\beta 1$ ) is a regulatory cytokine participating in the production and deposition of extracellular matrix in various tissues, including joint cartilage. The aim of the present study was to compare the concentrations of transforming growth factor $\beta 1$ in canine

\footnotetext{
${ }^{1}$ Escuela de Medicina Veterinaria, Sede Concepción, Universidad Santo Tomás, Chile

${ }^{2}$ Centro Veterinario Alemán Kleintierklinik, Concepción, Chile

${ }^{3}$ E-mail: cfischer@santotomas.cl
}

Recibido: 28 de octubre de 2017

Aceptado para publicación: 22 de marzo de 2018 
synovial fluid with ( $\mathrm{n}=12)$ and without DJD ( $\mathrm{n}=11)$, determined by an ELISA test. The mean concentration of TGB- $\beta 1$ in dogs without DJD was $16.8 \pm 7.1 \mathrm{ng} / \mathrm{ml}$, while in dogs with DJD it was $10.3 \pm 3.5 \mathrm{ng} / \mathrm{ml}(\mathrm{p}<0.05)$.

Key words: transforming growth factor $\beta 1$; synovial fluid; canines

\section{INTRODUCCIÓN}

La Enfermedad Degenerativa Articular (EDA) es la artropatía no inflamatoria más frecuente en los seres humanos y animales; es una alteración de las articulaciones móviles y desde el punto de vista macroscópico se caracteriza por la fragmentación y pérdida del cartílago articular y radiográficamente por estrechamiento del espacio articular, esclerosis del hueso subcondral, remodelación ósea, osteofitos en los márgenes articulares y entesiofitos en el sitio de inserción de tejidos blandos (Ettinger y Feldman, 2002). Las causas primarias de esta enfermedad se producen en animales que han realizado actividad física en exceso, asociado con el envejecimiento. La EDA secundaria es mucho más frecuente y se produce como secuela postraumática o seguida a desórdenes en la estabilidad. Como consecuencia de estas causas se producen cambios biomecánicos y bioquímicos que alteran los componentes de la articulación. Comienza con un deterioro del cartílago articular, con fisuras y pérdidas cartilaginosas, especialmente en zonas donde sufren mayor presión, para luego producirse sinovitis, esclerosis del hueso, engrosamiento de la cápsula articular y formación de osteofitos (Brusa y Boccia, 2000).

La sinovia es un líquido dializado del plasma de color transparente o amarillento, altamente viscoso por su contenido de ácido hialurónico y glucoaminoglicanos producidos por las células sinoviales de la articulación (Wittwer, 2012). Este líquido baña las estructuras articulares en el interior de la cavidad sinovial. Sus principales funciones son la nutrición y lubricación del cartílago articular
(Chico et al., 1995); asimismo, participa en la eliminación de desechos provenientes del metabolismo de los condrocitos (Richardson, 1982).

Los factores de crecimiento son un conjunto de sustancias de naturaleza peptídica, cuya misión es la comunicación intercelular a nivel molecular. Son capaces de modificar las respuestas biológicas celulares, ya que regulan la migración, proliferación, diferenciación, y metabolismo celular, e incluso la apoptosis (Peñarrocha et al., 2001). En condiciones fisiológicas, las plaquetas liberan el factor de crecimi ento transformante $\beta 1$ (TGF- $\beta 1$ ), entre otros, que modula la inflamación y la reparación de heridas (Sutter $e t$ al., 2004, Argüelles et al., 2006).

En Medicina Humana existen pocos estudios que reportan la concentración del TGF- $\beta 1$ en líquidos sinoviales presentes en artropatías. Fava et al. (1989) reportaron los casos de nueve pacientes con artritis reumatoide, cuatro con osteoartritis, dos con gota y uno con necrosis avascular, cuyas concentraciones de TGF- $\beta$ en los fluidos sinoviales fueron de $10.1,3.8$ y $8.0 \mathrm{ng} / \mathrm{ml}$ y sin actividad detectable, respectivamente. No se han encontrado estudios relacionados con concentración del TGF- $\beta 1$ en líquido sinovial y la comparación de este factor en caninos con y sin enfermedad degenerativa articular.

El objetivo de esta investigación fue comparar la concentración de TGF- $\beta 1$ en el líquido sinovial, en caninos con y sin enfermedad degenerativa articular, para una mejor comprensión del proceso de la degeneración articular en perros. 


\section{Materiales y Métodos}

El presente estudio se realizó en el Hospital Veterinario de la Universidad Santo Tomás, sede Concepción, ubicado en la ciudad de Concepción, VIII región, Chile. Se utilizaron 23 caninos adultos, sin distinción de sexo, ni raza, los cuales fueron divididos en dos grupos (grupo 1, sin EDA [n=11]; grupo 2, con EDA [ $\mathrm{n}=12]$ ). El criterio de selección para el grupo 1 fue que no presentasen alteraciones en el hemograma y estar clínicamente sanos al momento de la toma de muestra; es decir, constantes fisiológicas normales como temperatura $\left(38.1-39.2^{\circ} \mathrm{C}\right)$, frecuencia respiratoria (10-30 resp/min), frecuencia cardíaca (60-160 lat/min) y tiempo de llenado capilar (1-2 s) (Mendoza, 2011), además de no presentar alteraciones ortopédicas y radiológicas articulares al momento de realizar el examen radiológico. En cambio, en los pacientes incluidos en el grupo 2 se confirmó un diagnóstico ortopédico y radiológico de EDA al momento de la recolección de sangre y de líquido sinovial.

El examen ortopédico consistió en evaluar al paciente en descanso, caminando, trotando y la reexaminación tras el ejercicio, además de pruebas ortopédicas como flexión, extensión y abducción de la articulación (Piermattei et al., 2007). Luego de realizar el examen ortopédico, se procedió a la evaluación radiográfica (Ecotron EPX-F3200 HF) en conjunto con un digitalizador (Carestream Vita Flex CR X-ray) con dos vistas de cada articulación, con el fin de determinar un deterioro del cartílago articular como fisuras y pérdidas cartilaginosas, especialmente en zonas donde sufren mayor presión, que pueden producir sinovitis, esclerosis del hueso, efusión articular y formación de osteofitos (Johnston, 1997). Con base a los signos radiológicos hallados, se determinó la presencia o ausencia de EDA (Impellizeri et al., 2000).
El líquido sinovial se extrajo de codo, cadera o rodilla, según la articulación afectada, con una jeringa de $10 \mathrm{ml}$ con aguja de $18 \mathrm{G}$, que se introdujo en la articulación para aspirar el fluido en forma aséptica (Figura 1). Se tuvo en cuenta que la presión negativa de la jeringa fuese liberada antes del retiro de la aguja de la articulación para evitar contaminación de la muestra (Figura 2). Esta fue depositada en un tubo con EDTA y centrifugada a $1500 \mathrm{rpm}(101 \mathrm{~g})$ por 5 minutos (Fischer et al., 2014). Para la determinación de TGF- $\beta 1$ se empleó la técnica cuantitativa de inmunoensayo enzimático con un kit comercial específico para caninos (Quantikine ${ }^{\circledR}$ ELISA, Mouse/Rat/Porcine/ Canine TGF $\beta 1$ Inmunoassay).

Para determinar si los datos presentaban distribución normal se utilizó la prueba de normalidad Shapiro-Wilk (S-W: $\mathrm{p}<0.05$ ). Al no poseer distribución normal se utilizó la prueba de Mann-Whitney para determinar diferencias estadísticas. Se aceptó un valor de $p<0.05$ como diferencia significativa al realizar la comparación entre grupos.

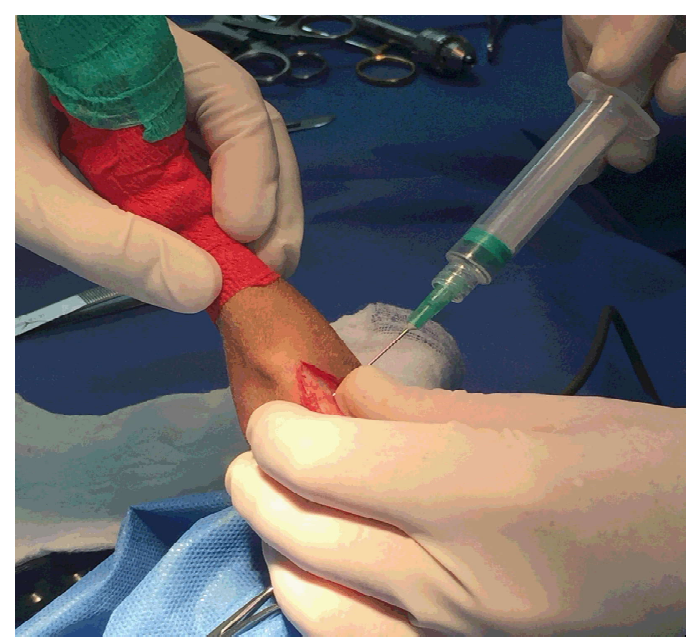

Figura 1. Extracción de líquido sinovial intraquirúrgico 


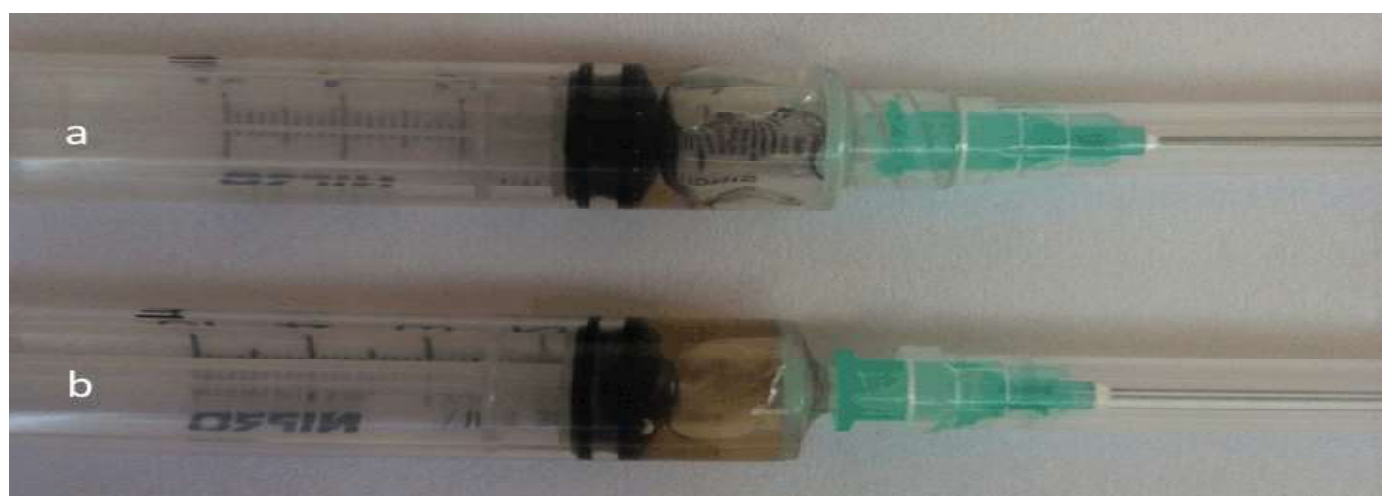

Figura 2. a: jeringa con líquido sinovial de articulación negativo a la enfermedad degenerativa articular (EDA). b: jeringa con líquido sinovial de articulación con EDA

Cuadro 1. Concentración del TGF- $\beta 1$ ( $\mathrm{ng} / \mathrm{ml})$ en el líquido sinovial de canes con $\mathrm{y}$ sin enfermedad degenerativa articular (EDA)

\begin{tabular}{ccc}
\hline Muestras & Sin EDA & Con EDA \\
\hline 1 & 19.87 & 7.19 \\
2 & 26.83 & 13.45 \\
3 & 12.42 & 16.32 \\
4 & 12.49 & 13.55 \\
5 & 11.97 & 10.58 \\
6 & 16.75 & 8.41 \\
7 & 31.49 & 11.98 \\
8 & 15.17 & 10.01 \\
9 & 17.45 & 8.75 \\
10 & 14.35 & 4.90 \\
11 & 6.30 & 13.21 \\
12 & & 5.60 \\
\hline Promedio & 16.82 & 10.33 \\
D.E. & 7.12 & 3.50 \\
\hline
\end{tabular}

\section{Resultados}

La concentración de TGF- $\beta 1$ en caninos con y $\sin$ EDA, según la prueba de ELISA, se muestra en el Cuadro 1. Los caninos sin
EDA presentaron una concentración de 16.82 $\pm 7.12 \mathrm{ng} / \mathrm{ml}$ (promedio \pm desviación estándar), mientras que en los caninos con EDA fue de $10.33 \pm 3.50 \mathrm{ng} / \mathrm{ml}(\mathrm{p}=0.01)$.

\section{Discusión}

La concentración media de TGF-âl para el grupo con EDA de $10.33 \mathrm{ng} / \mathrm{ml}$ fue significativamente menor que aquella en canes $\sin$ EDA $(16.82 \mathrm{ng} / \mathrm{ml})$, lo que demuestra lo indicado por Fava et al. (1989), que ante la presencia de una enfermedad degenerativa articular existe daño en la matriz del cartílago (Mc Ilwraith, 1982) y, con ello, lesiones en los condrocitos, los cuales son los que sintetizan la mayor parte de los TGF- $\beta 1$.

Los condrocitos afectados tienen reducidas sus capacidades proliferativas (Dominice et al., 1986), lo cual indica que con la presencia de EDA se pierde el equilibrio entre el anabolismo y catabolismo del cartílago (Aspden et al., 2001), perdiéndose estructuras fundamentales como la matriz extracelular del cartílago (Blanco et al., 1998), el cual tiene funciones de contención importante para el condrocito (Sánchez, 2008). 


\section{Conclusiones}

Los perros que presentan enfermedad degenerativa articular tienen una menor concentración de TGF- $\beta 1(10.32 \mathrm{ng} / \mathrm{ml})$ que perros sanos $(16.62 \mathrm{ng} / \mathrm{ml})$.

\section{Literatura Citada}

1. Argüelles D, Carmona JU, Pastor J, Iborra A, Viñals L, Martínes P, Bach E, Prades M. 2006. Evaluation of single and double centrifugation tube methods for concentrating equine platelets. Res Vet Sci 81: 237-245. doi: 10.1016/j.rvsc.2005.12.008

2. Aspden RM, Scheven BA, Hutchison JD. 2001. Osteoarthritis as a systemic disorder including stromal cell differentiation and lipid metabolism. Lancet 357: 1118-1120. doi: 10.1016/ S0140-6736(00)04264-1

3. Blanco FJ, Guitian R, VázquezMartul E, De Toro FJ, Galdo F. 1998. Osteoarthritis chondrocytes die by apoptosis. A possible pathway for osteoarthritis pathology. Arthritis Rheum 41: 284-289. doi: 10.1002/1529. 0131 (199802) $41: 2<284::$ AID ART12>3.0.CO;2-T

4. Brusa M, Boccia F. 2000. Enfermedad articular degenerativa canina: consideraciones sobre el manejo médico terapéutico ¿Son los condroprotectores una alternativa? Analecta Vet 20(1): 5-13.

5. Chico AC, Martí JM, Durall N. 1995. Artrocentesis y análisis del líquido sinovial en pequeños animales. Clín Vet Pequeños Anim 15: 135-144.

6. Dominice J, Levasseur $C$, Larno $S$, Ronot X, Adolphe M. 1986. Age-related changes in rabbit articular chondrocytes. Mech Ageing Dev 37: 231-240.

7. Ettinger S, Feldman E. 2002. Medicina interna veterinaria. Buenos Aires: Intermédica. $2181 \mathrm{p}$.

8. Fava R, Olsen N, Keski-Oja J, Moses H, Pincus T. 1989. Active and latent forms of transforming growth factor beta activity in synovial effusions. J Exp Med 169: 291-296.

9. Fischer C, Troncoso I, Luzio A, Opazo Á, Rios C, Cherres M, Ionita JC. 2014. Concentración de factor de crecimiento transformante beta 1 obtenido con dos métodos distintos de centrifugación en plasma de perros. Hosp Vet 6: 105-109.

10. Impellizeri JA, Tetrick MA, Muir P. 2000. Effect of weight reduction on clinical signs of lameness in dogs with hip osteoarthritis. J Am Vet Med Assoc 216: 1089-1091.

11. Johnston SA. 1997. Osteoarthritis: joint anatomy, physiology, and pathobiology. Vet Clin North Am Smal Anim Pract 27: 699-723.

12. Mcllwraith CW. 1982. Current concepts in equine degenerative joint disease. J Am Vet Med Assoc 180: 239249.

13. Peñarrocha M, Sanchís B, Martínez G. 2001. Factores de crecimiento y proteínas que influyen en el crecimiento óseo: aplicaciones en implantología oral. Periodoncia 11: 205-216.

14. Piermattei D, Flo GL, DeCamp CE. 2007. Manual de ortopedia y reparaciónde fracturas en pequeños animales. $4^{\mathrm{a}}$ ed. St. Louis, Missouri: Saunders. 836 p.

15. Richardson DW. 1982. Function and pathology of synovial fluid. Proc $28^{\text {th }}$ Annual Convention of the American Association of Equine Practitioners. Atlanta, Georgia, USA.

16. Sánchez Naranjo JC. 2008. Fisiología del condrocito articular. Rev Colomb Reumatol 15:21-33.

17. Sutter WW, AJ Kaneps, AL Bertone. 2004. Comparison of hematologic values and transforming growth factor- $\beta_{1}$ and insulin-like growth factor concentrations in platelets concentrates obtained by use of buffy coat and apheresis methods from equine blood. Am J Vet Res 65: 924-930.

18. Wittwer F. 2012. Manual de patología clínica veterinaria. Valdivia, Chile: América. $200 \mathrm{p}$. 